\title{
Test rig for rod seals contact pressure measurement
}

\author{
G. Belforte ${ }^{1}$, M. Conte $^{2}$, L. Mazza $^{1}$, T. Raparelli ${ }^{1}$ \& C. Visconte ${ }^{1}$ \\ ${ }^{1}$ Department of Mechanics, Politecnico di Torino, Italy \\ ${ }^{2}$ Manufacturing Processes Department - Tribology Unit, Tekniker, Spain
}

\begin{abstract}
The sealing efficiency and the extent of the friction force in an elastomeric seal counterpart sliding contact depend highly on the contact characteristics at the interface. In particular, a deep knowledge of the interface contact pressure is needed.

The work herein described deals with the design of a new test bench for an indirect contact pressure measurement. In particular, the radial force exerted by a rod seal sliding on a sensor-equipped portion of a cylinder rod is measured; during the seal displacement, the contact area between the seal and the sensorequipped rod increases, so that the radial force trend during time can be acquired. Taking the sliding velocity at a low constant value, contact pressure can be indirectly computed by differentiating the force signal. This way it is possible to perform measurements without introducing a third body between the seal and its counterpart, so avoiding any modification of the contact surface.

Initially, the test bench working principle is described, highlighting all critical aspects related to the radial force transmission from the seal to the load sensor; subsequently, the test bench requirements were defined. Finally, the design solution was presented.
\end{abstract}

Keywords: contact pressure, radial force measurements, seals, elastomers, pneumatics.

\section{Introduction}

Analyzing seals performances is a particularly complex and time consuming goal. In fact, there is a wide variability in terms of available materials, geometries and dimensions; furthermore, the system behaviour is highly 
influenced by working conditions, that is by applied loads, acting pressure, greasing or lubrication, temperature and velocity. In particular, a deep knowledge of the contact characteristics at the rubber-metal interface is required, since both sealing efficiency and the extent of the friction force depend on contact pressure.

A number of numerical and experimental studies aimed at evaluating contact pressure between a seal and its counterpart were reported. Bignardi et al. [1] analytically computed contact pressure between a pneumatic lip seal and a cylinder rod using experimental data from photoelastic tests. Yang and Salant [2] developed an analytical procedure involving contact pressure calculation in order to evaluate the behaviour of a tandem reciprocating hydraulic rod seal. Misiewicz et al. [3] employed a piezoelectric pressure mapping system to measure contact area and contact pressure between an agriculture tyre and the soil, obtaining accurate distribution at the contact surface. Lee et al. [4] employed film sensors for measuring contact area and maximum contact pressure on a lip seal for hydraulic rotating shafts, under different interference fits. Pressure sensitive films were also employed for static measurements of local contact pressure in pneumatic applications by Conte [5] and by Belforte et al. $[6,7]$; it was seen that these sensors were effective in firstly estimating the contact characteristics (contact pressure and contact area). Nevertheless, a limit of film sensors is due to their non-negligible thickness and to the difficulty of positioning them between the two parts in contact [8].

Some other authors reported non intrusive experimental methods. An ultrasonic technique was used by Marshall et al. [9] in a bolted connection, with the advantage of actual contact conditions determination. Poll et al. [10] developed a test bench for indirect contact pressure measurement. The radial force exerted by a seal mounted on a sensor-equipped rod was recorded over time and contact pressure was obtained by differentiating this signal; the rubberlike material viscoelastic influence was minimized, because there was nearly no additional deformation of the seal during the measurement. This radial force meter required a force sensor with a special design and no pressure load could be applied to the seal.

The work herein presented refers to the design and manufacturing of a new test bench for contact pressure measuring on a rod lip seal for pneumatic actuators (rod diameter $=20 \mathrm{~mm}$ ). The test bench is based on the same working principle described in [10] but adopts different design solutions, with the aim of minimising as much as possible additional friction forces that would affect the measurements. In particular, cheaper load sensors were used; in fact, the test bench was equipped with two common uniaxial load cells instead of suitably designed sensors. Furthermore, the test bench configuration allows a pressure load to be applied on the seal during the measurement.

In the following, the test bench working principle was firstly described. Subsequently, all critical aspects related to radial force transmission from the seal to the load sensor were discussed and test bench requirements were defined. Finally, the test bench design solution was presented. 


\section{Measuring principle and test bench requirements}

The experimental test consisted in acquiring over time the radial force that a lip seal exerts on a sensor-equipped portion of a cylinder rod, while a rod seal slid on it at constant velocity. The sensor-equipped rod can be obtained splitting the rod into two sectors, a sensor-equipped part and a fixed parte.

The contact area was scanned during the seal motion. Figure 1 shows the seal at the beginning of the test (a), during the measurement phase (b), i.e. when the seal is gradually engaging the sensor-equipped rod, and at the end of the test (c).

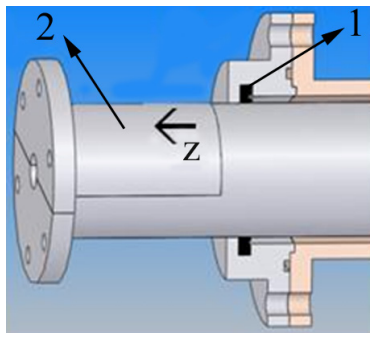

a)

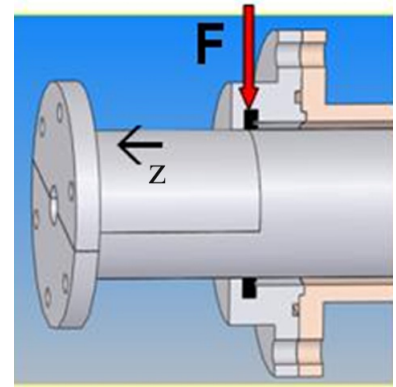

b)

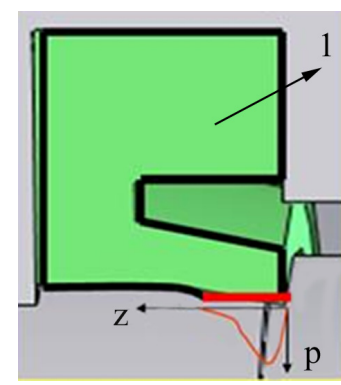

c)

Figure 1: $\quad$ Measuring sequence.

Since the seal (1) gradually slides in $z$ direction on the sensor-equipped part of the rod (2), a gradual increase of the contact area occurs.

Consequently, an increasing of the radial force $F$ can be detected, due to the exerted contact pressure, until a final force value is reached; this value corresponds to a complete contact of the lip seal, of length L, with the sensorequipped rod. Thanks to the constant velocity motion, it is possible to correlate the time-radial force trend with the $z$ direction-contact pressure $p$ trend along the contact surface. In fact, contact pressure distribution between the seal and the counterpart can be worked out differentiating the acquired force signal. With reference to Figure 2, representing a portion of the sensor-equipped rod (diameter $D$ ), the measured force $F$ can be worked out as the resultant of the contact pressure $p$ distribution over the semi-rod circular surface.

The balance equation (1) along the $y$ direction is:

$$
d F(z)=d z \cdot 2 \cdot \int_{0}^{\frac{\pi}{2}} p(z) \cdot \frac{D}{2} \cdot \sin \alpha \cdot d \alpha
$$

By integrating, it will be obtained:

$$
d F(z)=d z \cdot p(z) \cdot D
$$

Dividing both terms of (2) by time dt, $\mathrm{p}(\mathrm{x})$ can be obtained as:

$$
p(z)=\frac{d F(z)}{d t} \cdot \frac{d t}{d x} \cdot \frac{1}{D}=\frac{d F(z)}{d t} \cdot \frac{1}{V \cdot D}
$$

where $V$ is the constant sliding velocity of the seal along the $z$-direction. 


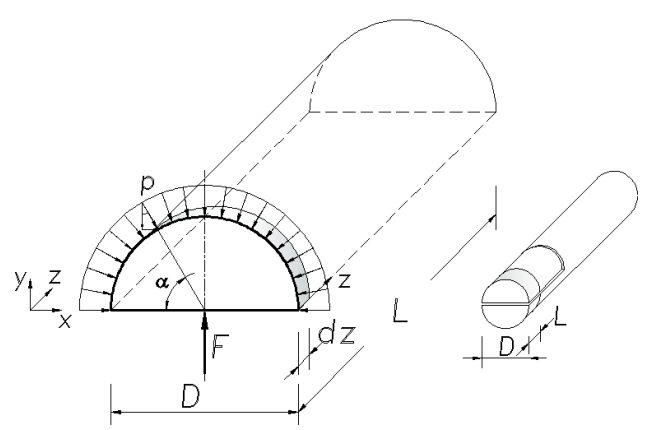

Figure 2: $\quad$ Sensor-equipped portion of the rod.

This measurement method reproduces actual working conditions without introducing additional deformations, so avoiding friction contribution due to the material viscoelastic behaviour; this characteristic represents a great advantage with respect to other experimental methods.

Measuring also the axial friction force exerted by the seal, the friction coefficient could also be determined.

In order to be able to measure the seal radial force as described previously, the cylinder rod must be cut in two portions, one of them connected to a load sensor. Figure 3 shows the chosen configuration, made up by: a fixed rod portion; a movable rod portion, integral with a plate for radial force transmission, denoted in the following as the "movable group".

A first requirement consisted of reproducing the rod nominal diameter, so to ensure nominal contact conditions between the seal and the rod. A gap $h_{l}$, with parallel surfaces A and B, was provided between the fixed and the movable rod portions in order to transmit the radial force, which tend to press the two portions one against the other. Values of $h_{l}$ in a range of $20 \div 50 \mu \mathrm{m}$ can be considered sufficient for allowing radial force transmission to a load cell with a defined stiffness and for avoiding the seal intrusion inside the gap. Another gap of height $h_{2}$ was provided between surfaces $\mathrm{C}$ and $\mathrm{D}$, in order to permit free (without friction) relative displacements. A pair of very low friction guide-bearings ensured guiding the movable group displacement along the seal radial force direction ( $y$-direction). In Figure 3, the seal radial force and the seal friction force are denoted as $F y$ and $F z$ respectively.

The seal friction force $F z$ is balanced by the reaction forces $R_{A}$ and $R_{B}$ of the low friction guides. The seal radial force $F y$ is balanced by the reaction force $R_{L}$ of a load cell placed between the movable group and the fixed frame (not shown in Figure 3). Since during the measurement $F y$ moves along the $z$-axis, $F y$ and $R_{L}$ act as a couple, causing a moment to arise on the movable group; the low friction guides also balanced this moment.

A low friction pad avoided the mobile group rotation about the $y$-axis, thus ensuring the correct relative position of the two portions of the rod, i.e. the rod cylindrical shape. 

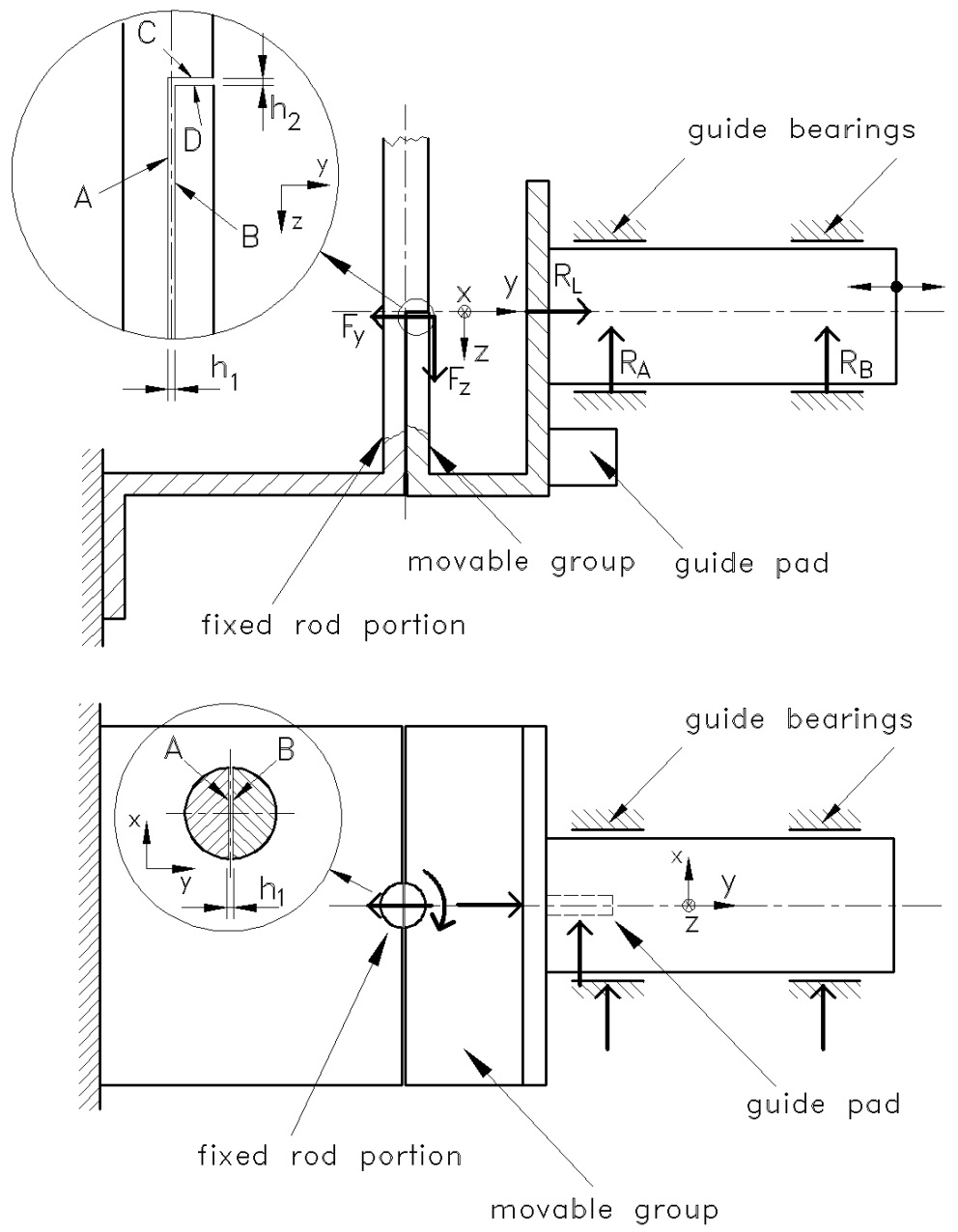

Figure 3: Test bench fuctional scheme: geometry requirements.

The load cell deformation must be less than the nominal gap $h_{l}$; to this aim, a load cell with a defined stiffness should be selected.

\section{Test bench structure}

A special test bench for an indirect measurement of contact pressure on a lip seal for pneumatic cylinder rod was designed and manufactured. It can reproduce actual working conditions. A vertical configuration was chosen, as shown in Figure 4. The bench can be divided into three main parts: the actuation part on the top, the measuring part at the bottom and the vertical fixed frame. 
The seal motion is driven by a linear electric actuator, whose velocity can be controlled within a range of $0,5-300 \mathrm{~mm} / \mathrm{s}$. The actuator is equipped with an antirotation system and with a wire-type sensor, for displacement and velocity recording.

A pneumostatic bearing (1) allows guiding the seal holder (2) linear motion without additional friction forces, to permit a regular seal displacement even at very low velocities. Connection between the linear bearing (1) and the driving actuator is provided by a universal joint (3) and a spacing collar (4).

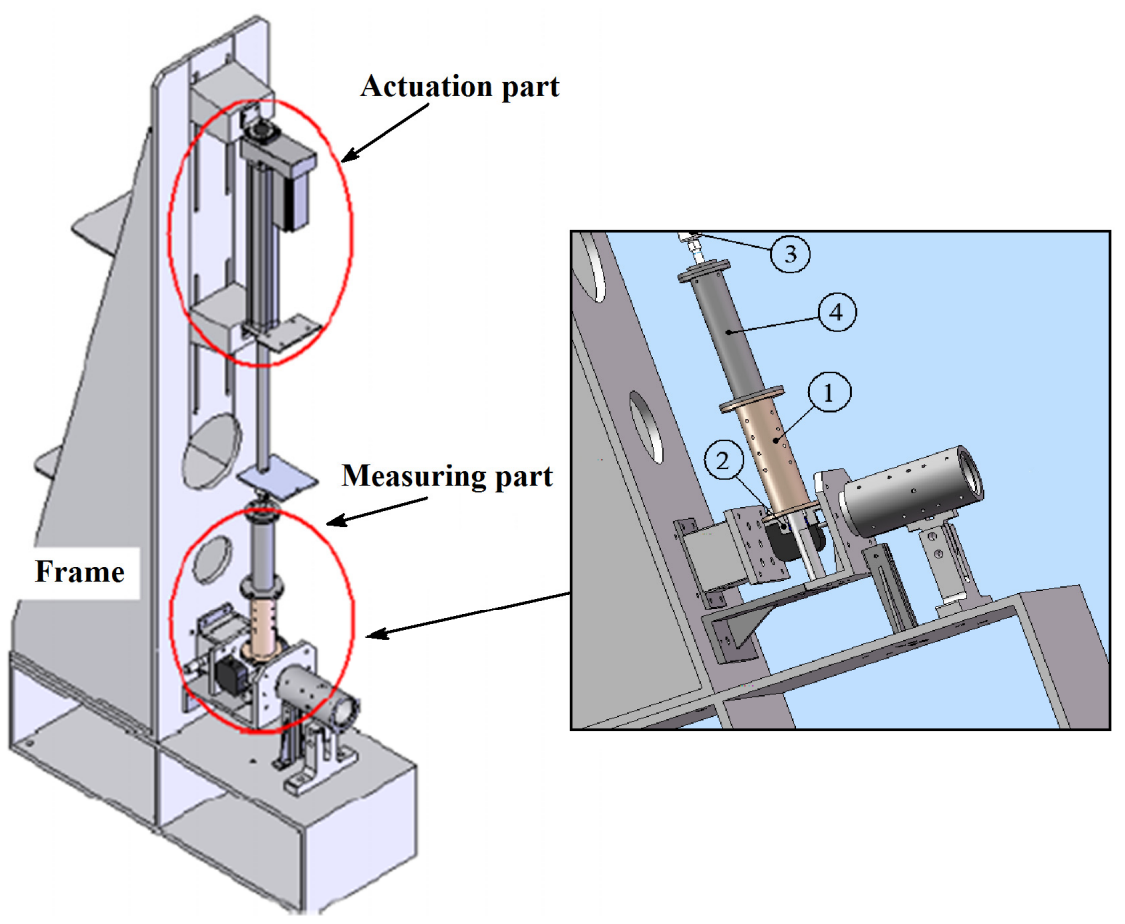

Figure 4: $\quad$ Test bench structure.

A detail of the test bench measuring part is shown in Figure 5. During the test, the seal is driven at first over the fixed part of the measuring rod (5) and then over the sensor-equipped rod portion (6). A couple of load cells (7), one of them shown in Figure 5, record the time-radial force trend during the seal motion. The movable group is supported by a pneumostatic bearing (8) and by a pneumostatic pad (9), with the aims described in the previous section. This way, a correct force transmission from the sensor-equipped rod to the load cells is ensured. Since a very precise relative positioning of the various components is required, a micro-positioner (10) and micro-metric screws (11) are used, together with calibrated metal shims. A nominal height of $50 \mu \mathrm{m}$ was chosen for the gaps $h_{1}$ and $h_{2}$; this value was considered a good trade-off between the need of good precision measurements and acceptable machining costs. 


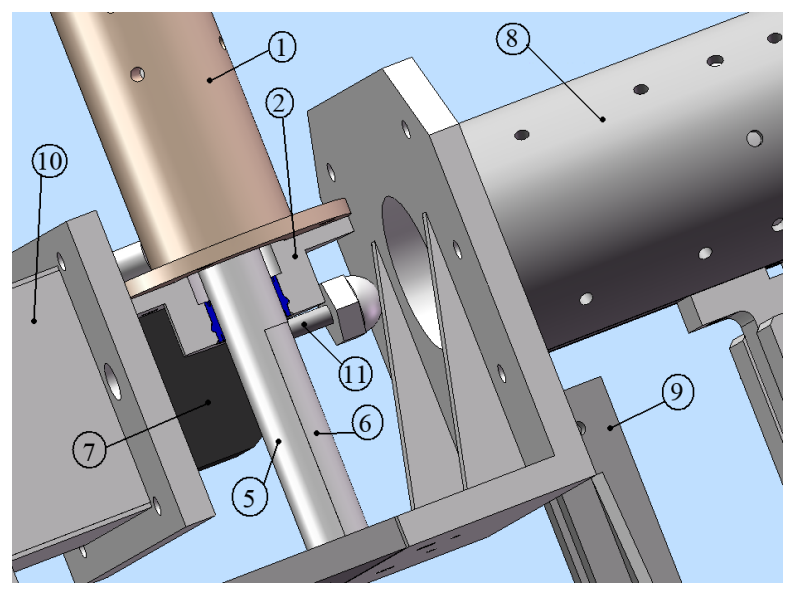

Figure 5: Details of the test bench measuring part.

The seal holder (2) is provided by a chamber that can be connected to the compressed air supply line, so that a pressure load can be applied on the seal. The chamber sealing is ensured on one side by the seal under test; on the opposite side, a small air leakage is allowed through a very narrow gap between the seal holder and the rod. Consequently, regulating the chamber supply pressure, it is possible to vary the seal pressure load.

\section{Conclusions}

A new test bench for an indirect contact pressure measurement between a pneumatic rod seal and its counterpart was designed. A rod seal is made sliding at slow constant velocity over a special rod, made up of a fixed portion and of a sensor-equipped portion; the latter allows recording during time, i.e. during the increase of contact area between the seal and the sensor-equipped rod, the radial force exerted by the seal on the rod. Differentiating in time this signal, it is possible to obtain indirectly the contact pressure distribution over the seal-rod contact surface.

The chosen measuring method has the great advantage of avoiding the interposition of sensors between the seal and the counterpart, so that the contact conditions are not modified; furthermore, a pressure load on the seal under test can be applied.

In order to perform the measurements correctly, any friction source different from the seal itself must be avoided. To this aim, pneumostatic components were provided and special design solutions were chosen.

A critical aspect is the test bench assembling: very narrow gaps must be provided between some parts and parts alignments must be done very carefully. To this aim, the test bench was provided by a precision linear positioner and micro-metric screws; calibrated metal shims were also used. 


\section{References}

[1] Bignardi, C., Manuello, A. \& Mazza, L., Photoelastic measurements and computation of the stress field and contact pressure in a pneumatic lip seal. Tribology International, 32(1), pp. 1-13, 1999.

[2] Yang, B. \& Salant, R. F., Numerical model of a tandem reciprocating hydraulic rod seal. Journal of Tribology, 130(3), pp. 032201.1 - 032201.7, 2008.

[3] Misiewicz, P. A., Richards, T. E., Blackburn, K. , Brighton, J. L., Hann, M. J. \& Godwin, R. J., Techniques for estimating contact pressure resulting from loaded agriculture tyres. American Society of Agricultural and Biological Engineers Annual International Meeting 2008, 1, pp. 521-535, 2008.

[4] Lee, C. Y., Lin, C. S., Jian, R. Q. \& Wen C. Y., Simulation and experimentation of the contact width and pressure distribution of lip seals. Tribology International, 39(9), pp. 915-920, 2006.

[5] Conte M., Interaction between seals and counterparts in pneumatic and hydraulic components. PhD Thesis, Politecnico di Torino, June 2009.

[6] Belforte, G., Conte, M., Manuello, A., Mazza, L. \& Visconte, C., Experimental and numerical evaluation of contact pressure in pneumatic seals. Tribology International, 42(1), pp.169-175, 2009.

[7] Belforte, G., Conte, M. \& Mazza L., Study on pneumatic cylinder piston seals behaviour. 15th International Sealing Conference, Stuttgart, Germany, 7-8 October 2008.

[8] Wu, J. Z., Herzog, W. \& Epstein M., Effect of inserting a pressensor film into articular joints on the actual contact mechanics. Journal of Biomechanical Engineering, 120, pp.655-659, 1998.

[9] Marshall, M.B., Lewis, R. \& Dwyer-Joyce, R. S., Characterisation of contact pressure distribution in bolted joints. Strain, 42(6), pp.31-43, 2006.

[10] Debler, C., Gronitzki, M. \& Poll, G., Investigation into the sealing contacts of reciprocating elastomeric seals-correlation of calculations with measurements and optical observations. 17th International Conference on Fluid Sealing, York, U.K., pp.169-186, 2003. 